\section{Vesículas extracelulares como predictores tempranos de diabetes gestacional}

\author{
MARIO ARIAS ${ }^{1, a}$, LARA J. MONTEIRO ${ }^{1, b}$, \\ STEPHANIE ACUÑA-GALLARDO ${ }^{1, \mathrm{c}}$, MANUEL VARAS-GODOY ${ }^{1,2, \mathrm{~b}}$, \\ GREGORY E. RICE ${ }^{1, \mathrm{~b}}$, MAX MONCKEBERG ${ }^{3}$, \\ PILAR DÍAZ ${ }^{3}$, SEBASTIÁN E. ILLANES ${ }^{1,3}$
}

\section{Extracellular vesicle concentration in maternal plasma as an early marker of gestational diabetes}

Background: During pregnancy, there is an increase in the amount of extracellular vesicles, especially placental exosomes, in maternal plasma. Aim: To isolate and characterize extracellular vesicles from blood during the three trimesters of pregnancy and to evaluate their capacity to identify patients at risk of developing gestational diabetes. Material and Methods: A case-control study was conducted in a cohort of 50 pregnant women with plasma samples taken in each trimester. Six women who developed gestational diabetes were paired with three healthy controls per case (a total of 19). Clinical characteristics were recorded at first prenatal appointment, and blood samples were obtained during the first, second and third trimesters. Extracellular vesicles were isolated from plasma by the commercial kit, ExoQuick ${ }^{\mathrm{TM}}$. Nanoparticle tracking analysis, was used to characterize the obtained extracellular vesicles. Results: The total concentration of extracellular particles isolated from maternal plasma increased along with gestational age. The size of the extracellular vesicles obtained in the first trimester of pregnancy was very similar between groups (144 $\pm 37 \mathrm{~nm}$ for controls and $143 \pm 34 \mathrm{~nm}$ for patients with gestational diabetes mellitus). Moreover, the concentration of extracellular vesicles collected in the first trimester, was significantly higher in patients who developed gestational diabetes mellitus later in pregnancy compared to normoglycemic pregnant women ( $7.94 \times 10^{8}$ and 5.15 $\left.x 10^{8}, p=0.03\right)$. Conclusions: Our results provide an insight into the potential capacity of first trimester plasma extracellular vesicles as early biomarkers for the prediction of gestational diabetes mellitus.

(Rev Med Chile 2019; 147: 1503-1509)

Key words: Biomarkers; Diabetes, Gestational; Extracellular Vesicle.

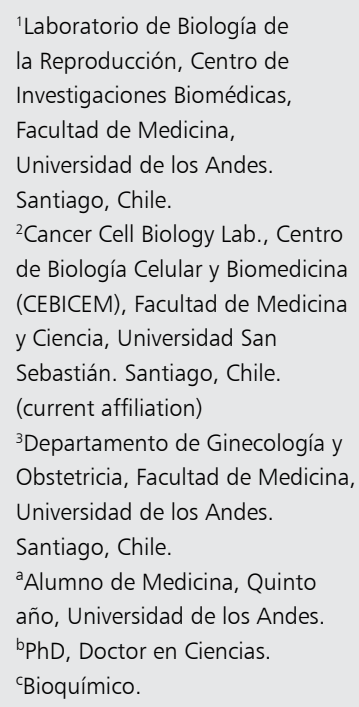

Trabajo no recibió financiamiento Los autores declaran no tener conflictos de interés

Recibido el 3 de julio de 2019, aceptado el 28 de noviembre de 2019.

\section{Correspondencia a:}

Sebastián E. Illanes, MD, MSc,

Universidad de los Andes

Centro de Investigaciones

Biomédicas

Laboratorio de Biología de la

Reproducción

Av. Plaza 2501, Las Condes,

7620001

Santiago, Chile.

sillanes@uandes.cl
L a diabetes gestacional (DG) se define como cualquier intolerancia a la glucosa con inicio o primer reconocimiento en el embarazo ${ }^{1,2}$. A nivel mundial, esta patología afecta aproximadamente $13 \%$ de las mujeres embarazadas ${ }^{3}$ y en Chile, puede alcanzar prevalencias de hasta
$17,7 \%{ }^{4}$. El pronóstico para los próximos años es que la prevalencia de DG incremente, asociado al aumento de la obesidad, una fecundidad más tardía y un aumento de la prevalencia de síndrome metabólico en mujeres de mayor edad, lo que se traducirá en una mayor carga asistencial, demanda 
por servicios especializados y mayores costos ${ }^{4}$.

La recomendación para el diagnóstico de DG en Chile es realizar el test de tolerancia con $75 \mathrm{~g}$ de glucosa oral (TTGO) entre las 24-28 semanas de gestación ${ }^{4}$. Aunque la prueba de TTGO es un test adecuado desde un punto de vista diagnóstico, presenta la gran desventaja de ser tardío en el embarazo. Por lo tanto, el feto y la madre están expuestos a un ambiente hiperglicémico durante la primera mitad del embarazo, que podría producir cambios epigenéticos en el feto y que explicarían el aumento del riesgo de obesidad y DM2 durante su vida adulta ${ }^{6}$. Es por esto que existe la necesidad de identificar biomarcadores que permitan identificar precozmente en el embarazo a las pacientes que desarrollarán DG.

Actualmente, hay estudios que han identificado biomarcadores plasmáticos asociados con el riesgo de desarrollo de DG, tales como el factor de crecimiento placentario, proteínas plasmáticas asociadas al embarazo y proteínas de unión a hormonas sexuales ${ }^{12-14}$. Un estudio reciente de nuestro laboratorio, evaluó la posibilidad de utilizar distintas proteínas plasmáticas maternas integradas en un algoritmo, al inicio del embarazo, para predecir que pacientes posteriormente desarrollaban DG. La curva ROC obtenida alcanzó un área bajo la curva de 0,870 con una sensibilidad de $81,4 \%$ y una especificidad de $80,0 \%{ }^{15}$. Sin embargo, a la fecha los estudios con biomarcadores predictores de DG aún carecen de la validez requerida para su uso en la clínica. Proponemos que el rendimiento para la detección temprana podría mejorarse con el uso de biomarcadores adicionales, como las vesículas extracelulares (VEs).

Las VEs se pueden clasificar en dos categorías principales: exosomas, con origen endosomal y tamaño entre 50-150 nm, y microvesículas, con origen en la membrana plasmática y entre 50-500 $\mathrm{nm}$ de diámetro ${ }^{16}$. Se ha establecido que durante un embarazo fisiológico hay un aumento de la cantidad de VEs, especialmente exosomas de origen placentario, encontradas en el plasma materno ${ }^{17,18}$.

Cabe destacar que estos biomarcadores presentan una serie de ventajas sobre los biomarcadores utilizados anteriormente, incluyendo la estabilidad en el almacenamiento y la protección contra degradación ${ }^{20,21}$, no obstante el método gold-standard para la extracción de exosomas incluye la ultracentrifugación, proceso que requiere de equipos especializados que no están disponibles en los laboratorios clínicos y que consume una cantidad significativa de tiempo. Sin embargo, el kit de precipitación, ExoQuick ${ }^{\mathrm{TM}}$ no requiere de equipo especializado, y con tiempos de extracción significativamente más bajos, podría ser una buena alternativa a la ultracentrifugación para ocupar en los laboratorios clínicos. De este modo, pretendemos investigar si las concentraciones de VEs extraídas con ExoQuick ${ }^{\mathrm{TM}}$ kit a partir del plasma de mujeres que desarrollarán DG, logran reflejar el mismo aumento que se ha demostrado a través del método gold-standard ${ }^{19}$, acercando así el potencial uso de VEs como biomarcadores de DG a su aplicación clínica.

\section{Material y Método}

\section{Diseño del estudio}

El estudio fue aprobado por el Comité de Ética de la Universidad de los Andes y se obtuvo un consentimiento informado por escrito de todas las pacientes antes del reclutamiento. Se realizó un estudio de casos-controles basado en una cohorte prospectiva realizada en la Unidad de Obstetricia y Medicina Fetal del Hospital Parroquial de San Bernardo en Santiago, Chile, en la que los casos y los controles fueron estratificados según el diagnóstico de enfermedad. Las pacientes fueron reclutadas en la primera visita prenatal. Después del reclutamiento, se registraron los antecedentes clínicos y obstétricos de las pacientes (edad materna, paridad, índice de masa corporal, primer peso de control y antecedentes de macrosomía). Las muestras de sangre periférica se recogieron en un tubo de recolección de sangre de citrato de sodio BD Vacutanier ${ }^{\circledR}(4,5 \mathrm{ml})$ en tres momentos: primer (6-12 semanas), segundo (22-24 semanas) y tercer trimestre (32-36 semanas). A las 24-28 semanas de gestación, las mujeres embarazadas fueron evaluadas para DG mediante la prueba de TTGO y se estratificaron retrospectivamente en dos grupos: DG y controles sanos. Los casos de DG se diagnosticaron de acuerdo con los criterios del Ministerio de Salud de Chile ${ }^{4}$, el TTGO de 75 $\mathrm{g}$ es positivo si la glicemia de ayunas es $\geq 100 \mathrm{mg} /$ dL y/o la glicemia a las $2 \mathrm{~h}$ postcarga es $\geq 140 \mathrm{mg} /$ $\mathrm{dL}$, tomadas a las 24-28 semanas. De la cohorte prospectiva inicial $(\mathrm{n}=50)$ todos los casos de DG $(n=6)$ se seleccionaron en el momento del diagnóstico de DG y el grupo de control $(n=19$; 
3 controles por cada caso) se seleccionó al azar utilizando la misma cohorte y se asignó por edad, estatus socioeconómico e índice de masa corporal. Se utilizaron como controles mujeres embarazadas con un feto sin condiciones médicas crónicas o complicaciones obstétricas. Los criterios de inclusión para la cohorte fueron: un embarazo único, edad gestacional menor a 14 semanas confirmada por ultrasonido. Los criterios de exclusión fueron: historial de diabetes o resistencia a la insulina; uso de metformina hasta un mes antes del embarazo; glicemia en ayunas $>105 \mathrm{mg} / \mathrm{dL}$ o una concentración de glicemia aleatoria $>200 \mathrm{mg} / \mathrm{dL}$ en el primer trimestre; abuso de alcohol o drogas; $y$ enfermedad psiquiátrica.

\section{Aislamiento de VEs de circulación materna}

Las VEs del plasma se aislaron por precipitación con el reactivo comercial ExoQuick ${ }^{\mathrm{TM}}$ (System Biosciences, CA, USA) de acuerdo con las recomendaciones del fabricante. Se centrifugaron $300 \mu \mathrm{L}$ de plasma en 3 ciclos: $12.000 \mathrm{~g}, 10.000 \mathrm{~g}$ y 10.000 g durante $5 \mathrm{~min}$ a $4^{\circ} \mathrm{C}$, extrayendo en cada ciclo el sobrenadante. Posteriormente, la fracción de plasma centrifugado se agregó a $63 \mu \mathrm{L}$ de reactivo ExoQuick ${ }^{\mathrm{TM}}$ y se incubó a $4{ }^{\circ} \mathrm{C}$ durante 30 min. Luego, el complejo plasma-ExoQuick ${ }^{\mathrm{TM}}$ se centrifugó a $1.500 \mathrm{~g}$ durante $30 \mathrm{~min}$ a $4{ }^{\circ} \mathrm{C}$ de modo de obtener el precipitado de VEs que posteriormente se suspendió en $100 \mu \mathrm{l}$ de PBS.

\section{Análisis de seguimiento de nanopartículas}

La distribución de tamaño y la concentración de VEs se obtuvo mediante análisis de seguimiento de partículas (NTA, del inglés Nanoparticle Tracking analysis) utilizando el instrumento NanoSight NS300 (Worcestershire, Reino Unido). Las VEs aisladas del plasma materno se diluyeron 1000x con PBS y se agregaron a la cámara microfluídica $\left(25^{\circ} \mathrm{C}\right.$ y viscosidad a $\left.0,9 \mathrm{cP}\right)$. Subsecuentemente se ajustaron los parámetros de potencia del láser (potencia de laser: 9 y duración de captura: $30 \mathrm{seg}$. Se capturaron 3 videos por muestra que posteriormente fueron procesados y analizados para evaluar la media y moda del tamaño de las VEs así como un número estimado de las partículas obtenidas.

\section{Análisis estadístico}

Se aplicó la prueba de normalidad D'Agostino-Pearson para evaluar si los valores surgen de una distribución gaussiana. Las comparaciones estadísticas se realizaron utilizando la prueba de Mann-Whitney. La significancia estadística se estableció en $\mathrm{p}<0,05$. Los datos se analizaron utilizando el software GraphPad Prism versión 6.0. Se calcularon la sensibilidad, la especificidad y los valores predictivos positivos y negativos ocupando el programa STATA v.14.2.

\section{Resultados}

\section{Características demográficas y clínicas de la población estudiada}

Las características clínicas de las pacientes en el momento del reclutamiento (11-14 semanas) se resumen en la Tabla 1. Tal como se planificó por los criterios de selección, con respecto a la

Tabla 1. Descripción de las características clínicas y demográficas de las pacientes sanas (grupo control) y de las pacientes que desarrollaron diabetes gestacional en sus embarazos (grupo DG), a las 11-14 semanas de gestación

\begin{tabular}{|lccc|}
\hline Variables & $\begin{array}{c}\text { Grupo Control (n = 19) } \\
\text { Mediana (p25 - p75) }\end{array}$ & $\begin{array}{c}\text { Grupo DG (n = 6) } \\
\text { Mediana (p25 - p75) }\end{array}$ & p value \\
\hline Edad materna (años) & $30(20-33)$ & $29(20-36)$ & 0,887 \\
\hline Talla $(\mathrm{m})$ & $1,57(1.53-1.63)$ & $1,54(1,50-1,57)$ & 0,170 \\
\hline Peso $(\mathrm{kg})$ & $73(59-78)$ & $59(46-76)$ & 0,197 \\
IMC (kg/m²) & $28,2(23,4-31,6)$ & $26,0(18,9-30,8)$ & 0,407 \\
\hline TTGO (glucosa en ayunas, $\mathrm{mg} / \mathrm{dL})$ & $65(61-73)$ & $80(73-98)$ & 0,013 \\
\hline TTGO (2 h postcarga, $\mathrm{mg} / \mathrm{dL})$ & $89(69-102)$ & $148(144-165)$ & $<0,0001$ \\
\hline
\end{tabular}

Los resultados son expresados mediante la mediana con el rango intercuartílico (P25-P75). Significancia estadística, p $<0,05$, Mann-Whitney Test. GD: Diabetes gestacional; m: metros; kg: kilogramos; IMC: Índice de Masa Corporal (kilogramos/metros cuadrados); TTGO: test de tolerancia a $75 \mathrm{~g}$ de glucosa oral (milígramos/decilitros); mmHg: milímetros de mercurio. 
edad materna y el índice de masa corporal no hay diferencias significativas entre las pacientes que desarrollaron DG durante sus embarazos y el grupo normoglicémico. Existe como era de esperarse, una diferencia significativa entre los valores del TTGO de ambos grupos, tanto en el valor de la glucosa en ayuna $(\mathrm{p}=0,013)$ como en el valor de $2 \mathrm{~h}$ post $75 \mathrm{~g}$ de glucosa oral $(\mathrm{p}<0,0001)$.

\section{Cuantificación de VEs en plasma durante el embarazo}

Para evaluar la liberación de vesículas extracelulares a lo largo del embarazo, las VEs de plasma materno, aisladas con ExoQuick ${ }^{\mathrm{TM}}$, se cuantificaron en el primer, segundo y tercer trimestre del embarazo en todos los casos y controles. El análisis de seguimiento de nanopartículas demuestra que las VEs aumentaron significativamente (ANOVA, $\mathrm{p}=0,007)$ con la progresión del embarazo. Durante el segundo y tercer trimestre, la concentración de VEs fue aproximadamente 1,8 y 2,3 veces más alta en comparación con las VEs en el primer trimestre del embarazo, respectivamente (Figura 1).

\section{Cuantificación de VEs en el primer trimestre de embarazo}

Para analizar si había diferencias en las VEs derivadas de plasma materno en el primer trimestre de embarazo entre los grupos estudiados (DG y controles), se analizó la distribución del tamaño de las VEs obtenidas con ExoQuick ${ }^{\mathrm{TM}}$. No hubo diferencias significativas en la distribución del tamaño de las VEs entre DG y controles, como lo demuestran las curvas gaussianas similares (144,4 \pm 37 para los Controles vs $143 \pm 34 \mathrm{~nm}$ para DG) y los gráficos de media y moda de distribución de tamaños (Figuras 2A, B, C). Sin embargo, la concentración de VEs en el primer trimestre es significativamente mayor en el grupo de pacientes que desarrollaron DG en el tercer trimestre (puntos negros) en comparación con el grupo de control (puntos blancos) (Figura 3A). De hecho, la concentración media de VEs fue 3 veces más elevada en el plasma obtenido de mujeres que desarrollaron posteriormente DG que en mujeres cuyos embarazos fueron normoglicémicos (valor de $\mathrm{p}=0,0302$ ) (Figura 3A). Igualmente se evaluó la posibilidad de utilizar la concentración de las VEs, al inicio del embarazo, para predecir que pacientes posteriormente desarrollaban DG. La curva ROC alcanzó un área bajo la curva de 0,7982 . El punto

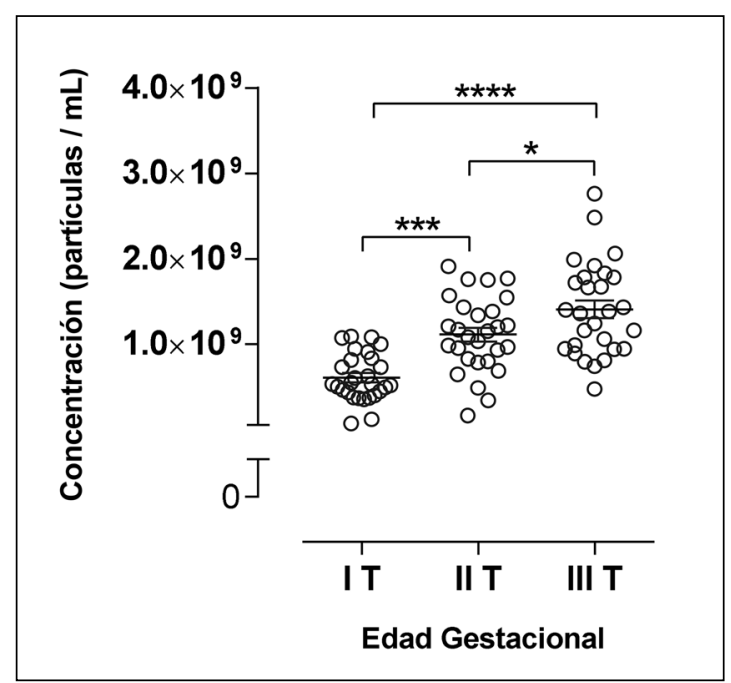

Figura 1. Perfil de VEs al largo del embarazo. El número de VEs se ha cuantificado en el plasma de las mujeres en el primer, segundo y tercer trimestre de embarazo de pacientes sanas y pacientes con diabetes gestacional $(n=25)$. Los resultados son el promedio \pm SEM. ${ }^{*} p \leq 0,05 ;{ }^{* * *} p \leq 0,001$; $* * * * p \leq 0,0001$. IT, primer trimestre; II , segundo trimestre; III T, tercer trimestre. VEs: vesículas extracelulares.

de corte óptimo de concentración de VEs para diagnóstico de DG fue de $5,745 \times 10^{8}$, con una sensibilidad del $83 \%$ y una especificidad del $74 \%$ (Figura 3B).

\section{Discusión}

El objetivo de nuestro estudio fue evaluar la capacidad de las VEs de identificar pacientes en riesgo de desarrollar diabetes gestacional, mediante procedimientos aplicables en clínica. Los datos obtenidos sugieren que a las 11-14 semanas las mujeres que desarrollarán DG, tienen concentraciones significativamente más altas de VEs que las mujeres que tienen un embarazo normoglicémico. Además, el análisis de seguimiento de nanopartículas demuestra que las VEs aumentan en concentración significativamente con la progresión del embarazo.

Estos resultados son concordantes con los obtenidos por Salomon ${ }^{17,18}$ a través de la extracción por ultracentrifugación y posterior análisis mediante el mismo sistema NTA, en donde se observa también un incremento progresivo de VEs durante el desarrollo del embarazo. Además, 


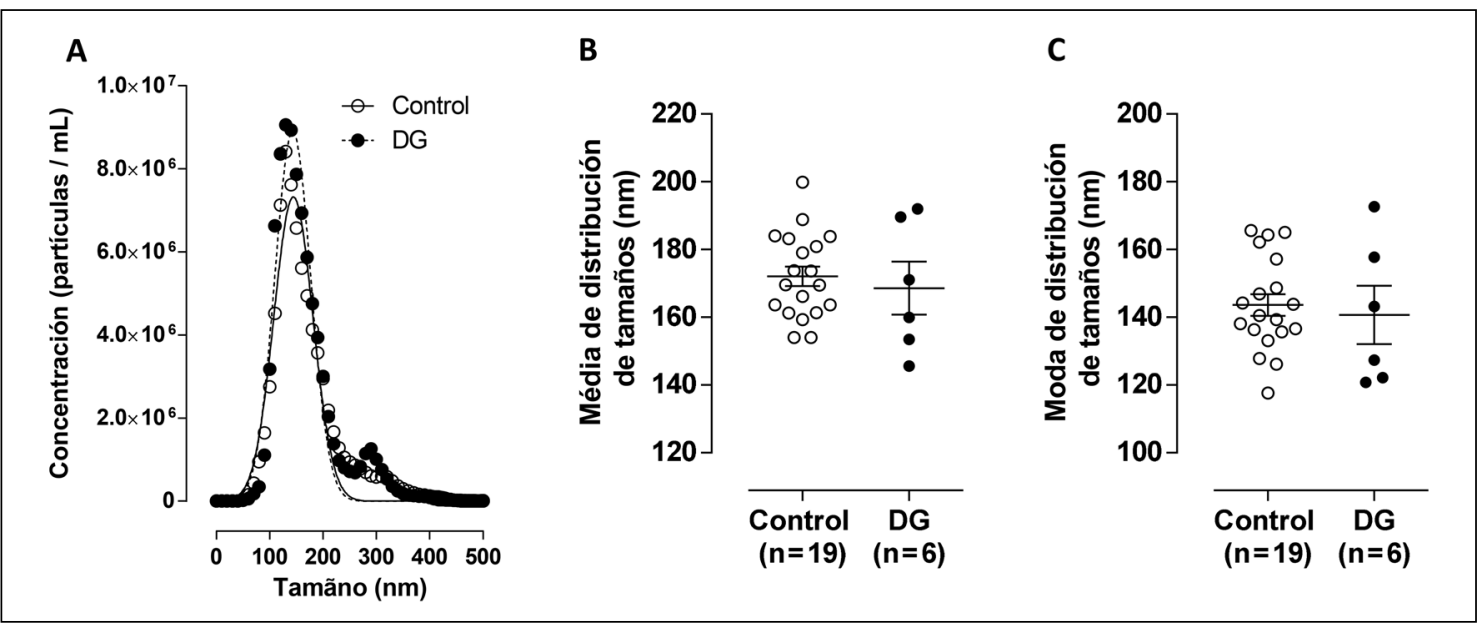

Figura 2. Análisis del seguimiento de partículas (NTA) de las VEs aisladas desde plasma materno en el primer trimestre de embarazo, utilizando ExoQuick ${ }^{\mathrm{T} M}$, en pacientes sanas y pacientes que desarrollaron DG. A. Distribución del tamaño de las VEs provenientes de plasma materno en el primer trimestre de embarazo. Los puntos negros corresponden a pacientes con DG y los puntos blancos a pacientes sanas. B. Media y C. Moda de distribución de tamaños de las VEs totales aisladas de los controles $(n=19)$ y DG $(n=6)$. (NTA, del inglés Nanoparticle Tracking analysis). VEs: vesículas extracelulares; DG: Diabetes gestacional.
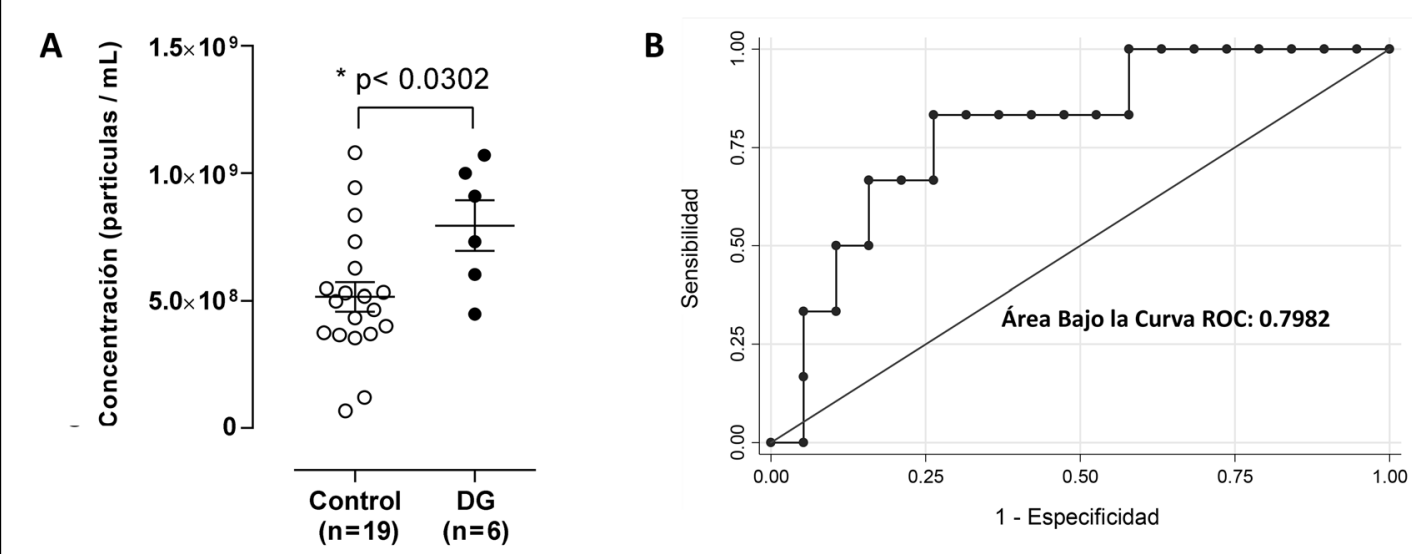

Figura 3. Concentración de Vesículas Extracelulares aisladas de plasma materno en el primer trimestre en controles y pacientes que desarrollaron DG. A: Concentración media de las VEs totales presentes en el plasma materno de los controles $(n=19)$ y DG $(n=6)$. Los resultados son la media \pm SEM. B: Curva ROC para la predicción de DG a través de la concentración de VEs en el primer trimestre de embarazo. VEs: vesículas extracelulares; DG: Diabetes gestacional.

en el estudio de Salomon se realizaron métodos adicionales para poder caracterizar estas VEs y definirlas como exosomas placentarios, analizando mediante microscopía electrónica la forma de las vesículas y se identificó la presencia de marcadores de membrana como PLAP y CD63 ${ }^{18}$. Nuestro método (mediante ExoQuick ${ }^{\mathrm{TM}}$ ) extrae vesículas extracelulares pequeñas enriquecidas en exosomas pero no de manera exclusiva. Sin embargo, sabemos que los exosomas son parte constituyente del grupo de partículas definidas como vesículas extracelulares pequeñas ya que tienen un diámetro que cae dentro de su intervalo de tamaño. De esta manera, se puede inferir que dentro de la 
población de VEs que se extrajo mediante el kit ExoQuick $^{\mathrm{TM}}$ existen exosomas presentes en las muestras obtenidas, además de otras vesículas.

El uso de VEs ricas en exosomas, extraídas mediante un método aplicable a la práctica clínica, es un potencial nicho para la implementación de un diagnóstico precoz de DG en la población. La importancia de la detección temprana radica en la posibilidad de intervenciones precoces para el tratamiento de esta patología. De esta manera, se evita el curso natural que supone exponer al feto durante las semanas en que no se ha logrado establecer el diagnóstico de DG a una hiperglicemia, generando una "programación fetal" mediada por cambios epigenéticos ${ }^{6}$, predisponiéndolo a largo plazo a patologías como obesidad, DM2 y en el caso de las mujeres, a desarrollar en futuros embarazos DG.

Se han propuesto otros modelos para lograr una predicción precoz de DG. Savvidou et al. utilizó datos clínicos (edad, edad gestacional, IMC, raza, antecedentes familiares de diabetes y GDM anterior) e incorporó HDL y t-PA al modelo multivariable para clasificar a mujeres con riesgo de desarrollar la patología ${ }^{22}$. Nanda et al. también combinó datos clínicos maternos con valores de adiponectina y $\mathrm{SHBG}^{23}$. También se han estudiado algunas proteínas de origen placentario como potenciales predictores de DG. Un estudio demostró que a las 11-14 semanas el factor de crecimiento placentario en plasma (PlGF, del inglés placental growth factor) podía predecir $63 \%$ de las mujeres que desarrollarían DG. De forma similar, cuando se combinan los niveles plasmáticos de PlGF con el peso y la edad materna, este valor predictivo aumenta a $78 \%{ }^{12}$. Un estudio reciente de nuestro laboratorio logró un modelo multivariable, utilizando colesterol total, triglicéridos, insulina, HOMA, lipoproteínas de baja densidad y activador tisular del plasminógeno (t-PA) obteniendo una sensibilidad y especificidad de alrededor de $87 \%{ }^{15}$. Sin embargo, ninguno de estos está validado para su uso en clínica, y existe consenso en la literatura de la necesidad de mejorar la sensibilidad y especificidad de estos algoritmos, pero sobre todo buscar biomarcadores que puedan ser medidos fácilmente en clínica, a un costo razonable.

Recientemente, se ha prestado especial atención a las VEs como biomarcadores para el diagnóstico y pronóstico de diferentes patologías ya que su caracterización y cuantificación propor- ciona información relevante acerca de alteraciones patológicas de sus células de origen ${ }^{20}$. Además, las VEs se pueden aislar fácilmente en diversos fluidos biológicos (plasma, orina, saliva) y son altamente estables, preservando su contenido biológico protegido de degradación ${ }^{20,21}$. Investigadores de nuestro grupo han demostrado que VEs y exosomas liberados de la placenta pueden ser detectados en el plasma materno a partir de las 6 semanas de gestación y que están aumentados en mujeres que desarrollarán $\mathrm{DG}^{19}$, demostrando que es posible utilizar estas VEs o marcadores exosomales para la predicción de DG durante el primer trimestre de embarazo. Sin embargo, para poder utilizar estos biomarcadores se necesita la introducción de métodos de laboratorio que puedan ser utilizados en el ambiente clínico. Nuestro trabajo demuestra que con el kit ExoQuick ${ }^{\mathrm{TM}}$, procedimiento sencillo, de bajo costo y rápido, se logra extraer VEs (ricas en biomarcadores exosomales), y que las concentraciones de éstas son mayores en pacientes que desarrollarán DG, confirmando lo que se había observado utilizando métodos más complejos. Estos resultados abren la posibilidad de la utilización de VEs como biomarcadores precoces de DG pudiendo utilizar estos valores dentro de modelos de predicción previamente estudiados.

Es importante señalar que un factor limitante en nuestro estudio es el tamaño de la muestra, sin embargo, durante el análisis de datos se obtienen resultados estadísticamente significativos, al igual que con el método de ultracentrifugación. Estos resultados a nivel piloto sientan la base para estudios posteriores con una muestra mayor, en donde se puede aplicar el kit comercial a un costo menor en comparación con el gold-standard, sin necesidad de equipamiento ni personal especializado, en una menor cantidad de tiempo ${ }^{21}$.

\section{Referencias}

1. ADA. 2. Classification and Diagnosis of Diabetes: Standards of Medical Care in Diabetes-2018. Diabetes Care 2018; 41 (Suppl 1): S13-S27.

2. ADA. 13. Management of Diabetes in Pregnancy: Standards of Medical Care in Diabetes-2018. Diabetes Care 2018; 41 (Suppl 1): S137-S43.

3. Melchior H, Kurch-Bek D, Mund M. The Prevalence of Gestational Diabetes. Dtsch Arztebl Int 2017; 114 (24): 412-8. 
4. MINSAL. Guía Perinatal 2015. Ministerio de Salud, Gobierno de Chile 2015; 1-466.

5. IADPSG, International Association of D, Pregnancy Study Groups Consensus P, Metzger BE, Gabbe SG, Persson B, et al. International association of diabetes and pregnancy study groups recommendations on the diagnosis and classification of hyperglycemia in pregnancy. Diabetes Care 2010; 33 (3): 676-82.

6. Monteiro LJ, Norman JE, Rice GE, Illanes SE. Fetal programming and gestational diabetes mellitus. Placenta 2016; 48 Suppl 1: S54-S60.

7. Clausen TD, Mathiesen ER, Hansen T, Pedersen O, Jensen DM, Lauenborg J, et al. High prevalence of type 2 diabetes and pre-diabetes in adult offspring of women with gestational diabetes mellitus or type 1 diabetes: the role of intrauterine hyperglycemia. Diabetes Care 2008; 31 (2): 340-6.

8. Damm P. Future risk of diabetes in mother and child after gestational diabetes mellitus. International journal of gynaecology and obstetrics: the official organ of the International Federation of Gynaecology and Obstetrics. 2009; 104 Suppl 1: S25-6.

9. Feig DS, Zinman B, Wang X, Hux JE. Risk of development of diabetes mellitus after diagnosis of gestational diabetes. CMAJ 2008; 179 (3): 229-34.

10. Ferrara A. Increasing prevalence of gestational diabetes mellitus: a public health perspective. Diabetes Care 2007; 30 Suppl 2: S141-6.

11. HAPO, Metzger BE, Lowe LP, Dyer AR, Trimble ER, Chaovarindr U, et al. Hyperglycemia and adverse pregnancy outcomes. N Engl J Med 2008; 358 (19): 19912002.

12. Eleftheriades M, Papastefanou I, Lambrinoudaki I, Kappou D, Lavranos D, Akalestos A, et al. Elevated placental growth factor concentrations at 11-14 weeks of gestation to predict gestational diabetes mellitus. Metabolism 2014; 63 (11): 1419-25.

13. Beneventi F, Simonetta M, Lovati E, Albonico G, Tinelli C, Locatelli E, et al. First trimester pregnancy-associated plasma protein-A in pregnancies complicated by subsequent gestational diabetes. Prenatal Diagnosis 2011; 31 (6): 523-8.

14. Caglar GS, Ozdemir ED, Cengiz SD, Demirtas S.
Sex-hormone-binding globulin early in pregnancy for the prediction of severe gestational diabetes mellitus and related complications. J Obstet Gynaecol Res 2012; 38 (11): 1286-93.

15. Correa PJ, Venegas P, Palmeiro Y, Albers D, Rice G, Roa $\mathrm{J}$, et al. First trimester prediction of gestational diabetes mellitus using plasma biomarkers: a case-control study. J Perinat Med 2019; 47 (2): 161-8.

16. van Niel G, D’Angelo G, Raposo G. Shedding light on the cell biology of extracellular vesicles. Nat Rev Mol Cell Biol 2018; 19 (4): 213-28.

17. Salomon C, Torres MJ, Kobayashi M, Scholz-Romero K, Sobrevia L, Dobierzewska A, et al. A gestational profile of placental exosomes in maternal plasma and their effects on endothelial cell migration. PLoS One 2014; 9 (6): e98667.

18. Sarker S, Scholz-Romero K, Pérez A, Illanes SE, Mitchell $\mathrm{MD}$, Rice GE, et al. Placenta-derived exosomes continuously increase in maternal circulation over the first trimester of pregnancy. J Transl Med 2014; 12: 204.

19. Salomon C, Scholz-Romero K, Sarker S, Sweeney E, Kobayashi M, Correa P, et al. Gestational Diabetes Mellitus Is Associated With Changes in the Concentration and Bioactivity of Placenta-Derived Exosomes in Maternal Circulation Across Gestation. Diabetes 2016; 65 (3): 598-609.

20. Revenfeld AL, Baek R, Nielsen MH, Stensballe A, Varming K, Jorgensen M. Diagnostic and prognostic potential of extracellular vesicles in peripheral blood. Clin Ther 2014; 36 (6): 830-46.

21. Yuana Y, Boing AN, Grootemaat AE, van der Pol E, Hau $\mathrm{CM}$, Cizmar P, et al. Handling and storage of human body fluids for analysis of extracellular vesicles. J Extracell Vesicles 2015; 4: 29260.

22. Savvidou M, Nelson SM, Makgoba M, Messow CM, Sattar N, Nicolaides K. First-trimester prediction of gestational diabetes mellitus: examining the potential of combining maternal characteristics and laboratory measures. Diabetes 2010; 59 (12): 3017-22.

23. Nanda S, Savvidou M, Syngelaki A, Akolekar R, Nicolaides KH. Prediction of gestational diabetes mellitus by maternal factors and biomarkers at 11 to 13 weeks. Prenat Diagn 2011; 31 (2): 135-41. 